\title{
The regulation of emerging technologies: is it possible to stem the tide?
}

\author{
Aliuska Duardo-Sanchez \\ ${ }^{a}$ Chair in Law and Human Genome Research Group \\ ${ }^{b}$ Department of Public Law, University of Basque Country, \\ 48940, Leioa Biscay, Spain
}

\begin{abstract}
.
Legal norms, generally, go very far behind social relations. By the time a norm comes to see the light, it is quite possible that the situation that it intends to regulate has changed and this - the norm - is outdated and ineffective from its very birth. Well, let us think the more difficult is the relationship between Law and emerging technologies; since, in order for them to be effectively regulated, it is necessary that there is a noticeable degree of anticipation on the part of the legislator. The task seems unfeasible, however, we believe that it is possible and essential to find, among the international legal acquis, a minimum standard establish the margins of action of scientific work, without suppose a brake for progress.
\end{abstract}

\section{Conceptual approach to emerging technologies.}

Gregory Day and Paul Shoemaker, in their book "Wharton on Managing Emerging Technologies" (New York: Wiley, 2000), describe emerging technologies such as those scientific innovations that can create a new industry or transform an existing one. The description includes discontinuous technologies derived from radical innovations, as well as more evolved technologies formed because of the convergence of previously separated research branches. It is quite common, therefore, to use the emerging or convergent adjectives, indistinctly. For our part, we prefer the term emerging technologies, which seems more graphic and appropriate for the reality that intended to be described, without even knowing that emerging technologies are, often, the result of the synergy of the different fields of scientific innovation. On the other hand, they are in all cases disruptive technologies, either by creating market niches offering new products or services, or by representing a break with the business model established in a particular sector.

Now, knowing when a certain technological innovation can be cataloged as an emerging technology, is almost as hard as anticipating its future consequences. By doing a quick search on the Internet, it is often found lists of very promising technologies for the upcoming times. Each institution, warehouse, specialized blog ... makes its own pool on what are the methodologies that will change, in a drastic way, the business model of one sector or another. The most current examples include zero-emission 
2

http://sciforum.net/conference/mol2net-04

automobiles that work with hydrogen; next generation robotics; genetic engineering techniques; developments in artificial intelligence; Nanotechnology; social networks, etc.

Its enormous complexity, its dizzying progress, and undoubtedly, the economic interests at stake, make it very difficult to anticipate the real impact of technological developments, especially as regards the risks and side effects for society. Aware of this fact, some international institutions and research groups have been showing their concern by dedicating studies that identify the risks and venture possible solutions. Thus, in the summer of 2013, the Council of Europe's Bioethics Committee (DH$\mathrm{BIO}$ ) invited the Rathenau Institute (Netherlands) to explore which emerging technologies could pose ethical and legal challenges from the perspective of dignity and human rights. The result was the report "From Bio to NBIC convergence - From Medical Practice to Daily Life", best known as the Ratheneau report. This document provides a perspective on emerging technologies, with the aim of clarifying what developments might require more attention from European institutions.

This study is done from the perspective of the NBIC Convergence; a concept that "is not neutral but very fruitful", according to the words of the drafters of the report, and that is based on the integration between the sectors of nanotechnology, biotechnology, information technology and cognitive sciences. The study shows that the NBIC convergence has resulted in a wave of emerging technologies that pose all kinds of ethical-social challenges, in aspects such as: security, privacy, autonomy, responsibility, physical and mental integrity, informed consent and access to technology. Also, in other lesser-known phenomena, such as human improvement (enhancement), ownership of biological data, freedom of information, consumer competencies and medicalization.

\section{Emerging technologies and fundamental rights}

It has been said many times that science and technology are neither perfect nor neutral. It would be naive to think that the technology sectors will regulate themselves, guiding themselves by ethical codes that will avoid possible adverse results, or sharing the benefits of scientific innovation. We believe that uncertainty, consubstantial with emerging technologies, should not be confronted by absolute fear or rejection; but from the responsibility and from the rights that have been universally recognized as goods, by seeking a life that is worthy for all beings human.

This is the perspective that underpins the Report on Ethical Issues Raised by Emerging Sciences and Technologies, developed for the Council of Europe's Bioethics Committee by Roger Strand and Matthias Kaiser, from the Center for Studies of Sciences and Humanities, University of Bergen, Norway, on January 23, 2015. The report focuses on the ethical implications of emerging technologies, taken Neurosciences, Nanotechnologies, and Information and Communication Technology as paradigmatic cases. In addition, three crosscutting aspects are discussed: 1) the diffused line between the medical and non-medical field, 2) the ethical question of world divisions and equitable access to medical advances and 3) the particular ethical challenges of use Military of the technologies.

Although, the report does not intend to touch the legal aspects, but only identify possible risks and threats of emerging technologies from an ethical perspective, they do so on the basis of human rights. Fundamentally, the rights reflected in articles 1, 2, 3 and 10 of the Oviedo Convention; and Articles 8 and 9 of the Convention for the Protection of Human Rights and Fundamental Freedoms, Signed in Rome on November 4, 1950. 


\section{Final Thoughts}

Technologies, emerging or not, have enormous potential for social change, for good and for evil. Identify which technologies keep the seed of social transformation; what are their possible benefits, how to ensure equitable access to them; what risks are involved and how to avoid or mitigate them legged the case- is undoubtedly one of the greatest challenges that we face in this century.

In this sense, from a legal point of view, two positions are held: on the one hand, from the reports analyzed point of view, the extension of the existing agreements to date, mainly the Oviedo Convention, to the treatment of emerging technologies would not suffice. From this particular vision, there is the urgent to establish a legal instrument of international scope and binding effects, which regulates the principles that should guide technological development, in general, paying special attention to emerging technologies, as these have the peculiarity of being little known, and therefore of more difficult prediction regarding its possible undesired implications. On the other hand, there are those who consider that the Oviedo Convention is a basic guide that has not yet shown its full effectiveness. An instrument that we already have and that we should take full advantage of, since the rights and interests that are sought to be protected are the same ones that would be affected by the indiscriminate use of emerging technologies.

In any case, what is clear is that the search for a minimum standard that establishes the margins of action of scientific-technical work must be done in accordance with widely accepted human rights traditions. 